\title{
Phase-locking of disordered two-dimensional Josephson junction arrays to microwave radiation
}

\author{
C. De Leo, ${ }^{*}$ G. Filatrella ${ }^{\dagger}$ and G. Rotoli ${ }^{\ddagger}$
}

August 30, 2000

\begin{abstract}
A connection between the disorder in the critical currents of two-dimensional arrays of Josephson junctions and the amount of phase-locking of a row to an external microwave signal is proposed. We have computed the probability of phase-locking as a function of the spread of the critical currents of the individual junctions. The analytic results are in good agreement with numerical simulations. The predictions can be also qualitatively compared to the experiments.
\end{abstract}

PACS. 85.25.Am, 85.25.Cp, 85.25.Na

Keywords: Supeconducting devices, Josephson arrays, Phase-Locking.

\section{Introduction}

Phase locking of Josephson Junctions (JJ) to external microwave is nowadays a standard tool to investigate the properties of such nonlinear oscillators. The basic idea is that by studying the phase lock to an external radiation one can infer on the possibility of spontaneous phase lock among the junctions. The importance of spontaneous phase lock is twofold: on one hand for applications because the power emitted by a single junction is not enough for many practical uses [1]. On the other hand, from a theoretical point of view, the phenomenon of entrainment of disordered oscillators has led to many important discoveries in nonlinear dynamics [2]. Experiments with external microwave irradiation have been performed [3] and explained for long Josephson junctions [4] and have been the starting point to understand the more complicated behavior of mutual phase-lock $[5,6]$. Series arrays of small Josephson junctions have also been investigated for many years until a recent result [7] has shown the underlying mathematical structure

\footnotetext{
*Dipartimento di Energetica, Università dell'Aquila, Località Monteluco I67040, L'Aquila, Italy e-mail:deleo@ing.univaq.it

${ }^{\dagger}$ Unità INFM Salerno and Facoltà di Scienze, Università del Sannio, Via Caio Ponzio Telesino, 11, I-82100 Benevento, Italy. e-mail: giofil@physics.unisa.it

‡Unità INFM and Dipartimento di Energetica, Università dell'Aquila, Località Monteluco I67040, L'Aquila, Italy email:rotoli@ing.univaq.it, CORRESPONDING AUTHOR
} 
that has allowed to better understand the conditions (and the difficulties) to achieve the phase-locked state. In Ref. [7] it has been shown that in the weak coupling limit JJ arrays coupled through a resonator can be mapped analytically onto the Kuramoto model, a general framework for such class of phenomena [8]. Two dimensional arrays of JJ have been proposed to achieve a better phase-locking among the junctions, when compared to series array. Unfortunately, it appears that some of the problems are still present in two-dimensional arrays. For instance Wiesenfeld, Benz, and Booi have shown that there is a transformation that completely decouples the $2 \mathrm{D}$ bare arrays in independent oscillators [1]. In spite of the simplifying assumptions used to perform the transformation (essentially to neglect disorder and inductive effects) a decoupling of $2 \mathrm{D}$ arrays in rows weakly interacting $[9,10]$ or not interacting [11] has shown good efficacy when compared with numerical simulations that include, to some extent, inductive effects.

The purpose of this work is to show that following the same scheme used in Ref. [11], namely to decouple the array in elementary oscillators constituted by rows, it is possible to predict the behavior of disordered 2D arrays irradiated by a microwave field. The results obtained have been compared with numerical simulations of disordered arrays with full mutual inductive effects. The behavior here described has been experimentally investigated with the use of the LTSEM technique [12]. We want to emphasize that also other studies indicate the relevance of the row decomposition. For instance in a recent experiment [13] it has shown that individual rows seem capable to phase-lock, so that the whole system is better described in terms of oscillators constituted by rows than by considering the individual junctions as elementary oscillators. Also in theoretical investigations, such as those of Barahona and Watanabe [14], the motion of fluxons across the arrays, in absence of microwave excitations, has been handled starting from the dynamics of the elementary rows.

The paper is organized as follows: in Section 1 we will describe the model and the basic calculations, in Section 2 we will compare the analytic results with numerical simulations, in Section 3 we discuss the 
question of the existence of a threshold for phase-locking of junctions in the array, finally in Section 4 we will discuss some relevant experimental consequences of our model.

\section{$1 \quad$ Statistical analysis}

A schematic drawing of a two-dimensional plane array is shown in Fig. $1, V_{i, j}$ and $H_{i, j}$ are the vertical and horizontal junctions connected to the node $i, j$, the terms $I_{C i, j}^{h}$ are the corresponding critical currents; here the indices $i=1, \ldots, N ; j=1, \ldots, M$ denote the mesh; $h=0,1$ indicates the direction of the branch, horizontal or vertical, respectively. For convenience we introduce also the dimensionless quantities $\delta_{i, j}^{h}$, they the values assumed by a random variable representing the spread in the critical currents around their expectation value $\bar{I}_{C}$ such that:

$$
I_{C i, j}^{h}=\bar{I}_{C}\left(1+\delta_{i, j}^{h}\right)
$$

The equations for an $N \times M$ array with overdamped junctions accounting also for the mutual inductances between the branches can be derived as follows. We first compute the current across a branch $I_{i, j}^{b}$ in terms of the Josephson relation between the phase across the junctions, $\varphi$. Normalizing the current respect to the expectation value of the junction critical currents $\bar{I}_{C}$ and the time respect to $\hbar / 2 e R I_{0}$ ( $R$ is the normal resistance of the junctions due to the quasiparticle tunneling) and in the overdamped limit, it reads $[15]$ :

$$
I_{i, j}^{h}=\left(1+\delta_{i, j}^{h}\right) \sin \varphi_{i, j}^{h}+\dot{\varphi}_{i, j}^{h},
$$

here, $\sin \varphi_{i, j}^{h}$ is the current through the Josephson element, and $\dot{\varphi}_{i, j}^{h}$ is the current through a resistor that mimics the quasi-particle terms; To satisfy the Kirchhoff law for the currents in each node we use the mesh current $I_{i, j}^{s}$ that are connected to the branch currents by the relationship (see Fig. 1):

$$
I_{i . j}^{h}=\delta_{1, h}\left(I_{i, j}^{s}-I_{i, j+1}^{s}+I_{e x t}\right)+\delta_{0, h}\left(I_{i, j}^{s}-I_{i+1, j}^{s}\right)
$$


$\delta_{i, j}$ is the Kronecker operator, $I_{e x t}$ denotes the normalized bias current injected in the top nodes and extracted from the bottom nodes. If there is an $a c$ bias added to the $d c$ bias the current term can be written as:

$$
I_{e x t}=I_{b}+I_{r f} \cos (\omega t)
$$

It is more convenient to use a vector $\overrightarrow{I^{b}}$ (whose components are the branch currents $I_{i, j}^{h}$, the LHS of Eq. (3) and a vector $\overrightarrow{I^{s}}$ (whose components are the mesh currents $I_{i, j}^{s}$ ) to rewrite in a matrix form Eq.s $(3,4)$ :

$$
\overrightarrow{I^{b}}=\hat{K} \overrightarrow{I^{s}}+\vec{\gamma}
$$

The fluxoid quantization rule for each mesh gives another set of equations, one for each mesh. Normalizing the flux respect to the elementary flux quantum $\Phi_{0}=h / 2 e$ and with the use of the SQUID parameter $\beta_{L}=2 \pi L^{\prime} I_{0} / \Phi_{0}\left(L^{\prime}\right.$ is the self-inductance of the SQUID), they read:

$$
\frac{1}{\beta_{L}} \Sigma \varphi=\phi^{T O T}
$$

where the sum $\Sigma$ spans over the junctions of a mesh, $\phi^{T O T}$ is the total flux in the mesh due to the external flux and to the field generated by the currents flowing in all meshes of the array. Eq.s $(3,6)$ can be cast into an implicit [16] or an explicit scheme, [17] (cf. this last reference for a discussion about the performances of both methods), including mutual inductance terms. The goal of our analysis is to predict for a given value of $\gamma_{r f}$ the average number of phase-locked junctions. The basic assumptions will be that the system can be decomposed in rows, and that either a whole row is phase-locked to the external microwave, or none of the junctions belonging to that row is. This is consistent with the experimental observation made in Ref. [12] and has also been analytically predicted by [10] for sufficiently small amount of disorder. The ac radiation on a single junction induces a Shapiro step; the current height $\Delta \gamma$ is [18]:

$$
\Delta \gamma=\frac{\gamma_{r f}}{\left(-{ }^{2}+1\right)^{1 / 2}}
$$


and the central point is on the unperturbed IV curve. To compute such unperturbed (i.e., without $r f$ ) curve in presence of disorder we use the same technique of Ref. [11]: we assume that each row for a given bias oscillates at a frequency $\omega$ determined by the row mean critical current:

$$
\gamma=\left[\omega^{2}+\frac{1}{M} \sum_{k=1}^{M}\left(1+\delta_{k}\right)^{2}\right]^{1 / 2} .
$$

(Here we indicate $\delta_{i, j}^{h}$ simply by $\delta_{k}$, where $k$ is an index spanning all the junctions in the array.) Note that being the $\delta_{k}$ random variables, $\gamma$ itself is a random variable, representing the bias at which a row should be fed so that it oscillates at the given frequency (or voltage) $\omega$. For sake of simplicity we assume the actual bias point of the array to be the center of the phase-locking step (as it was done in the experiments [12]), i.e., the point where the unperturbed IV of the whole array oscillates at the frequency - of the external microwave, even for zero amplitude of the external drive [see Eq. (8)]. The bias point is therefore the expectation value, i.e., $\bar{\gamma}=I_{b} / \bar{I}_{C}$, so at the center of the step is simply (if we assume that the terms $\delta_{k}$ to have zero expectation value, i.e., $\bar{\delta}=0$, then in the normalization units here adopted the expectation value of critical current is 1$)$ :

$$
\bar{\gamma}=\left(\omega^{2}+1\right)^{1 / 2}
$$

To achieve voltage-locking of a single row to the frequency $\omega$ of the external microwave, the bias should be:

$$
\gamma_{\omega}=\left[\omega^{2}+\frac{1}{M} \sum_{k=1}^{M}\left(1+\delta_{k}\right)^{2}\right]^{1 / 2}
$$

So, for a given set of the parameters, a row will be phase-locked to the external microwave if the random bias $\gamma_{\omega}$ will be within the extension of phase-locking range, respect to its expectation value. This happens with a probability $P_{p l}$, when the following condition is satisfied:

$$
P_{p l}=\mathcal{P}\left(\left|\bar{\gamma}-\gamma_{\omega}\right|<\frac{1}{2} \frac{\bar{\gamma}_{r f}}{\left(\omega^{2}+1\right)^{1 / 2}}\right)
$$

where $\mathcal{P}$ represents the specific distribution law of the random variables appearing in the parenthesis and to evaluate the phase-locking range we use again its expectation value obtained using $\bar{\gamma}_{r f}=I_{r f} / \bar{I}_{c}$. 
Inserting Eq.s $(7,10)$ into condition $(11)$ and expanding to the lowest order in the random variable $\delta_{k}$ we obtain:

$$
P_{p l}=\mathcal{P}\left(\gamma_{r f}>\left|\frac{2}{M} \sum_{k=1}^{M} \delta_{k}\right|\right)
$$

We make the further assumption that the $\delta_{k}$ are uniformly distributed in the interval $[-\epsilon, \epsilon]$. The interpretation of Eq. (12) is then that for a given distribution of the critical currents in a row (a collection of $\delta_{k}$ ) the row is phase locked to the external microwave if the amplitude of the microwave satisfies the condition (12). To handle this condition for relatively large values of $M$ we follow the same method used in Ref.[11], applying the central limit theorem we get that distribution of the deviation from the average value of the $\delta_{k}$ will be a Gaussian random variable, say $\xi$, with standard deviation $\sigma=2 \sigma_{0} / \sqrt{M}, \sigma_{0}$ being the standard deviation of the distribution of the $\delta_{k}$. For uniform distribution one gets $\sigma_{0}=\epsilon / \sqrt{3}$. A similar treatment can be also made when we assume a Gaussian distribution of the $\delta_{k}$, in this case $\xi=\frac{2}{M} \sum_{k=1}^{M} \delta_{k}$ is a Gaussian variable with standard deviation equal to $2 \epsilon / \sqrt{M}$ where $\epsilon$ here is the standard deviation of original Gaussian distribution.

Now let say that we irradiate the array with a given amplitude of the $\operatorname{rf}$ signal $\gamma_{r f}$, the Eq. (12) becomes:

$$
P_{p l}=\mathcal{P}_{\text {uniform }}\left(|\xi|<\gamma_{r f}\right)=\operatorname{Erf}\left(\gamma_{r f}\right)-\operatorname{Erf}\left(-\gamma_{r f}\right)=\frac{1}{\sqrt{2 \pi} \sigma} \int_{-\gamma_{r f}}^{+\gamma_{r f}} \exp \left(\frac{-\xi^{2}}{2 \sigma^{2}}\right) d \xi
$$

where

$$
\sigma=\sigma_{\text {uniform }}=\frac{2 \epsilon}{\sqrt{3 M}}
$$

Similarly for the Gaussian case we have:

$$
\sigma=\sigma_{\text {gauss }}=\frac{2 \epsilon}{\sqrt{M}}
$$

The number of rows phase locked will simply be on average $N P_{p l}$, and the probability distribution binomial. The case $M=2$ for uniform distribution can be better handled taking a slightly different analysis: For this low value of $M$ it is better to assume a Simpson distribution, i.e. the convolution of 
two uniform distribution involved, rather than a Gaussian distribution. In all simulations shown below the theory with $M=2$ refers always to Simpson rather than Gauss distribution when an uniform distribution of currents is assumed.

\section{Numerical results}

If our analysis is correct the average fraction of the phase locked rows for a given $r f$ power should be proportional to the difference of cumulative Gaussian distribution (error integral), so the predictions of Eq.s $(13,15)$ have been checked simulating Eq.s (2-6) using an explicit scheme similar to that used in [17]. Typical simulations are made as follows: random critical currents are extracted from both a uniformly distribution of amplitude $2 \epsilon$ and a Gaussian distribution with standard deviation $\epsilon$. The number of phase locked rows for a given value of the $r f$ power is counted after a typical transitory time $T=400$ normalized units, then the experiment is repeated with a new realization of random critical currents extracted from the same distribution. Finally the average fraction of phase locked rows is reported as a function of the amplitude of the external current drive $\gamma_{r f}$ (i.e. the square root of the microwave power). In Fig.2 are reported the main results of this paper, in all cases the statistical error associated with the points shown in the figures range from $5 \%$ to $15 \%$ (for sake of readability we do not report error bars in the figures). Simulations are reported for $N=8,16$ and 20, for some values of $M$ for both the uniform and Gaussian cases. We note that in general the numerical experiments agree with the theoretical estimates, especially for low values of the disorder. Moreover a good reproduction of theory is always achieved below $80 \%$ of locked junctions. When the curve reaches saturation, eventually the disagreement becomes more evident. An important factor is however the row number $N$ and its ratio with column number $M$ : for $N=8$ and $M=2,4,8$ the theory is well reproduced but systematic deviations are evident especially for the two largest $M$ cases (see Fig.2a); for $N=16$ a very good agreement is reached indicating that higher row numbers have a better statistics as can be expected (see Fig.2b,2d). However, if the number of columns becomes large, as is shown in Fig.2c for $M=12,16$ the agreement becomes worse, especially for 
values of rf-amplitude near saturation. A further increase in row number, $N=20$, again implies a better agreement (compare. again Fig.3c). Between the reasons of disagreement we quote: 1) the estimates of Eq. (13) are correct only to the first order in $\epsilon$; 2) the assumption that phase-locking occurs whenever the random bias of one row is within the phase-locking range respect to average IV is only approximate; 3) the assumption of simultaneous phase locking of all junctions in the row is only approximate, this is true especially in the case of arrays with relatively few row. Second order terms are however rather small (about 1\% for the data collected in Fig.2) so this cannot be a relevant source of disagreement. Point 2) can be a more relevant source of disagreement, but by increasing row number the statistics will improve, as is shown by difference between Fig.2a and 2b,2d. The last point deserves more attention: from simulation we see that in a disordered array simultaneous phase-locking of all junctions belonging to the same row is more difficult for arrays with $N \lesssim M$ because row coupling is probably not sufficient to overcome disorder along the row to reach phase-locking over the whole array. Despite the fact that rows are supposed statistically independent in the above model, an inspection of the number of phase-locked rows in the simulations shows that when few phase-locked rows occurs, the array seems unable to lock the remaining not phase-locked rows. This behavior is also more evident near saturation because the set of possibly "good" rows to be phase-locked with the increase of signal amplitude will exhaust and the remaining statistically "bad" rows cannot be locked.

We should point out that the difficult point to compare the numerical data with the experiments is to estimate $\gamma_{r f}$, because the power delivered to the array cannot be easily computed. A possible calibration might be done measuring the height of the Shapiro steps and then using Eq. (7). Another relevant characteristic of the experiment is the observed linearity between the applied power and the square of the number of the phase-locked junctions. In our figure this should correspond to a linear relationship between the rf-current and the number of phase-locked rows. This linear behavior is compatible with Eq. (12) or (13), when fluctuations due to the specific realization of the statistics are included. In fact 
one more difficulty to compare the theory with the experiments is that the theory predicts the average properties of the arrays, while the experiments are obviously performed on single realizations (as will be discussed in more detail in the next section).

In Fig. 3 we have compared the effect of the discreteness parameter $\beta_{L}$ on the system: as a general rule the array behavior follows the statistical model with a good accuracy if $\beta_{L}$ ranges from 0.5 to 1.5 . For low values of $\beta_{L}$ the hypothesis of independent rows cannot be satisfied because all junctions in the arrays become more tightly coupled, so we expect some deviations from the above theory in the numerical simulations, as in Fig.3(a) in the case of $\beta_{L}=0.2$ with the uniform distribution (similar result is obtained for Gaussian case). In any case also for such low $\beta_{L}$ values the saturation part of the curve is well reproduced by statistical approach and maximum observed deviation is about $20 \%$. When mutual inductances are included it has been found that the effect is to decrease the amplitude of the induced Shapiro steps predicted by Eq. (7) [16]: The amplitude of the Shapiro steps shows a minimum around $\beta_{L} \simeq 4$ [17]. This should also be reflected in the capability of disordered arrays to lock to an external source, as shown in Fig.3(a), where the numerical behavior significantly departs from the theoretical one for values of $\beta_{L}$ approximately higher than 1.5. We presume that for the $\beta_{L} \simeq 4$ case the assumption that all the junctions in each row are phase-locked, used to derive Eq. (8), fails. In fact for high values of $\beta_{L}$, i.e. low coupling, this is not anymore true, and this is also evident in Fig.3(b), where the numerical behavior for extremely high (and presumably unphysical) values of the discreteness parameter clearly shows a deviation. Nevertheless up to $\beta_{L}=100$ there is a monotonic increase of the number of phase-locked rows, in agreement with the re-increase of the phase-locking region [17].

In Fig. 4 we have checked the prediction of our theory for others values of the amplitude of the distribution $\epsilon_{\text {uniform }}$ for the uniform distribution case and standard deviation $\epsilon_{\text {gauss }}$. As expected since Eq. (10) is a linear expansion, the higher $\epsilon$ the worse the agreement between the numerics and the theory. 


\section{Threshold}

In the experiment of the Tübingen group [12] a threshold in the minimal amplitude of the external drive to phase-lock a row was observed. The authors ascribed the effect to a "laser type" behavior of superradiant arrays. Also in the experiment of Barbara et al. [13] it has been found evidence of threshold effects. The appearance of a threshold can be also ascribed to other effects, for instance noise in the system can prevent the appearance of a step before a certain amplitude of the external drive, thus introducing a threshold in Eq. (7) [20]. Yet another possibility is that the very disorder in the critical currents gives rise to a threshold. We want to propose a mechanism that can lead to the appearance of a threshold and is connected with the number of activated oscillators. For the underdamped junctions experiment [13] an explanation in terms of detuning between the natural frequencies of the oscillators and the cavity has been proposed [19], underlying that it is possible that the strong interaction between the cavity and the oscillat ors is responsible for the sudden increase of the number of phase-locked oscillators once a critical number of oscillators is activated. In the system under consideration the analysis is greatly simplified by the fact that the dynamics of the forcing term (the $r f$ field) is independent of the dynamics of the oscillators. To analyze the effect of the disorder we can simply assume that when too few rows are active it can just happen that there are not rows whose bias point $\gamma$ is close enough to the frequency of the external drive, so none of them can be phase-locked.

One more way is to look at what happens if one simulates one experiment at a time, and, to the contrary of the previously presented simulations, does not average over many realizations the number of phase-locked rows. So, we report here the average number of rows that must be activated before at least one is phase-locked at the external field. We underline that this procedure is more suitable for a comparison with the observation of a threshold in a specific sample. Summarizing, it is possible that for a given realization and for sufficiently small values of the driving current it is not possible to phase-lock

any row. When the drive increases, the minimal value at which a row can be phase locked and this value 
of the $r f$ drive will result to be the "threshold".

A quantitative analysis of the effect of disorder on the threshold can be performed as follows: the probability to find a value of the threshold $\gamma_{r f}^{\text {threshold }}$ is the probability to not find any row capable to be locked, out of the $N$ rows of the array, i.e.:

$$
P\left(\forall \text { rows }|\xi|>\gamma_{r f}\right)=\left[1-\frac{1}{\sqrt{2 \pi} \sigma} \int_{-\gamma_{r f}}^{+\gamma_{r f}} \exp \left(\frac{-\xi^{2}}{2 \sigma^{2}}\right) d \xi\right]^{N}
$$

A similar equation can be written also in the Gaussian distribution case. It is clear that such threshold goes quickly to 0 for large samples, and disappears in the thermodynamic limit $(N \rightarrow \infty)$. The numerical simulations of the array dynamics are compared to Eq. (16) in Fig.5. From this analysis and from simulations it is clear that the threshold thus obtained is very small compared to the one observed by Lachenmann et al. [12]. In fact a rough comparison can be done assuming that $\gamma_{r f}$ is proportional to the square root of the applied power, and then calibrating the amplitude to the power necessary to phase-lock all the junctions. Doing so, one obtains that the threshold is about $50 \%$ of the saturation current, a value that for an array $10 \times 10$ and an estimated standard deviation of the critical currents of $3 \%$ [12] is simply unrealistic: the probability to fabricate such array is negligibly small. We conclude that presumably the noise effects are dominant for the specific experiment [12].

\section{Conclusion}

We have showed that the decomposition of two-dimensional arrays in rows is an effective technique to predict the behavior of some disordered arrays irradiated by microwave. With this technique we were able to connect quantitatively the parameters of the disorder (essentially, the standard deviation of the random critical currents) with the number of rows phase locked to an external microwave source. A further practical use of this result might be to estimate the disorder in an array (a quantity that cannot be estimated easily for $2 \mathrm{D}$ arrays) by doing experiments as those performed by [12]. Unfortunately the

estimate is limited by two factors: i) the amount of current actually flowing into the array $\left(\gamma_{r f}\right.$ in our 
notation) can be estimated only roughly; ii) The relation is true in average, the results of the single measurements can deviate significantly, following a binomial distribution.

In the approach proposed an important factor in determining the response of disordered arrays to external signal is the ratio between column number $M$ and row number $N$ : arrays with $N \gtrsim M$ will phase-lock all their rows faster and easily to an external signal. If confirmed by further experimental work this can be an important information for the design of optimal array based oscillators. We note that in the experiment performed by Barbara et al. [13] arrays are precisely of the type $N \gtrsim M$.

The measurements of [12] took advantage of the unique properties of the LTSEM technique to directly visualize the phase-locked rows. In the more recent experiment by Barbara et al. [13] on underdamped arrays placed on a resonator and without external drive it was possible to carry out a similar investigation taking advantage of the fact that the junctions were underdamped (so from the simple inspection of the IV it was possible to estimate the number of active oscillators). Although similar in the spirit, it seems that the theoretical analysis here proposed is very different for the one proposed for the latter experiment [19]. Finally, both experiments have shown a threshold laser-like behavior. We have been able to reproduce a threshold only due to disorder, by analyzing the finite sample response. The estimate for the threshold seems to be unrealistically small when compared to the threshold experimentally observed. Still we believe that there might be cases of entrainment of disordered oscillators to an external drive where a threshold could appear as the result of finite number of oscillators.

\section{Acknowledgments}

We thank P.Carelli, M.Cirillo, G. Costabile, A.Petraglia, K.Wiesenfeld for useful discussions and suggestions. We gratefully acknowledge financial support from MURST COFIN98 project "Dynamics and Thermodynamics of vortex structures in superconductive tunneling". 


\section{References}

[1] K. Wiesenfeld, S.P. Benz and P.A.A. Booi, J. Appl. Phys. 76, 3835 (1994).

[2] S.H. Strogatz, "Norber Wiener's Brain Waves", in Lecture Notes in Biomathematics 100 (Springer, New York, 1994).

[3] G. Costabile, R. Monaco, and S. Pagano, J. Appl. Phys. 63, 5406 (1988).

[4] M. Salerno, M.R. Samuelsen, G. Filatrella, S. Pagano, and R.D. Parmentier, Phys. Rev. B41, 6641 (1990).

[5] R. Monaco, N. Grønbeck-Jensen, and R.D. Parmentier, Phys. Lett. A 151, 195 (1990).

[6] G.Filatrella, G.Rotoli, N.Grønbeck-Jensen, N.F.Pedersen, R.D.Parmentier, J. Appl. Phys. 72, 3185 (1992).

[7] K. Wiesenfeld, P. Colet, and S.H. Strogatz, Phys. Rev. Lett. 76, 404 (1996); Phys. Rev. E 57, 1563 (1998).

[8] Y. Kuramoto, in Lecture Notes in Physics, Vol. 39 (H. Araki ed., Springer, p. 420 (1975).

[9] G. Filatrella and K. Wiesenfeld, J. Appl. Phys. 78, 1878 (1995).

[10] A.S. Landsberg, Y. Braiman and K. Wiesenfeld, Phys. Rev. B 52, 15458 (1995).

[11] K. Wiesenfeld, A. Landsberg, G. Filatrella, Phys. Lett. A 233, 373 (1997).

[12] S.G. Lachenmann, T. Doderer, and R.P. Huebener, Phys. Rev. B 52, 14541 (1996).

A. Laub, M. Keck, T. Doderer, R.P. Huebener, T. Treuble, R. Dolata, T. Weimann, and J. Niemeyer, J. Appl. Phys, 83, 5302 (1998).

[13] P. Barbara, A.B. Cawthorne, S.V. Shitov, and C.J. Lobb, Phys. Rev. Lett. 82,

[14] M. Barahona and S. Watanabe, Phys. Rev. B57, 10893 (1998). 
[15] A.Barone and G.Paternò, Physics and Applications of the Josephson effect, Wiley 1982.

[16] J.R. Phillips, H.S.J.V. der Zant, J. White, T.P. Orlando, Phys. Rev. B 50, 9387 (1994).

[17] G. Filatrella, A. Petraglia, and G. Rotoli, Eur.Phys. J. B 12, 23 (1999).

[18] N. Grønbeck-Jensen and M.R. Samuelsen, Phys. Lett. A 191, 57 (1994).

[19] G. Filatrella, N.F. Pedersen, and K. Wiesenfeld, Phys. Rev. E 61, 2513-2518 (2000).

[20] G. Costabile, private communication.

\section{Figure Captions}

Fig. 1. The electrical scheme of $2 \mathrm{D}$ arrays.

Fig. 2. The dependence of the fraction of phase locked junctions as a function of the external $r f$ current. Symbols refer to simulations and solid lines to the theoretical predictions derived by Eq.(13), dot ted lines between symbols are draw as guide for eye. (a) The case of $M=2(\triangle), 4(\bigcirc), 8(\square)$ horizontal junctions for an $N=8$ array with uniform distribution; (b) the case of $M=2(\triangle), 4(\bigcirc), 8(\square)$ horizontal junctions for $N=16$ array with uniform distribution; (c) the case of $M=12$ horizontal junctions for an $N=16$ $(\diamond), 20(\nabla)$ array in the uniform distribution case; (d) the case of $M=2(\triangle), 4(\bigcirc), 8(\square)$ horizontal junctions for an $N=16$ array in the Gaussian distribution case. The parameters of the simulations are: $\beta_{L}=1, \epsilon_{\text {uniform }}=0.3, \epsilon_{\text {gauss }}=0.1, \omega=1.0, \bar{\gamma}=1.9$.

Fig. 3. The dependence of the fraction of phase locked junctions as a function of the external $r f$ current. The data refer to different values of the coupling parameter $\beta_{L}$. In (a) we show the lower values $\left(\beta_{L} \leq 2\right)$ and in (b) the higher values $\left(\beta_{L} \geq 2\right)$. The solid lines represent the predicted behavior derived by Eq.(13) that does not account for the $\beta_{L}$ dependence, dotted lines between symbols are draw as guide for eye. The parameters of the simulations are: $N=16, M=8, \epsilon_{\text {uniform }}=0.3, \omega=1.0, \bar{\gamma}=1.9$. The symbols shows the following values of $\beta_{L}: 0.2(\diamond), 1.0(\bigcirc), 1.5(\square), 2.0(\nabla), 5.0(\triangle), 10(\times), 100(+)$. Some 
curves is shown also for the Gaussian case with $\epsilon_{\text {gauss }}=0.1$, the values of $\beta_{L}$ in this case are: 1.5 ( $), 5$

\section{$(\mathbf{\Delta})$.}

Fig. 4. The dependence of the fraction of phase locked junctions as a function of the external $r f$ current.

(a) The data refer to different values of the distribution amplitude $\epsilon_{\text {uniform }}$ for the uniform distribution $\epsilon_{\text {uniform }}=0.1(\square)$ and $0.2(\bigcirc)$; (b) the data refer to different values of the standard deviation $\epsilon_{g a u s s}$ for the gaussian distribution $\epsilon_{\text {gauss }}=0.05(\square)$ and $0.15(\bigcirc)$. The solid lines represent the theoretical prediction of Eq.(13), dotted lines between symbols are draw as guide for eye. The parameters of the simulations are: $N=16, M=8, \omega=1.0, \bar{\gamma}=1.9$.

Fig. 5. The dependence of the probability to find a threshold as a function of the external $r f$ current. Symbols refer to simulations for $N=3$ (circle) and $N=8$ (squares); solid lines represent the behavior predicted by the theory Eq.(16), dotted lines between symbols are draw as guide for eye. The parameters of the simulations are: $M=3, \epsilon=0.3, \beta_{L}=1, \omega=1.0, \bar{\gamma}=1.9$. 


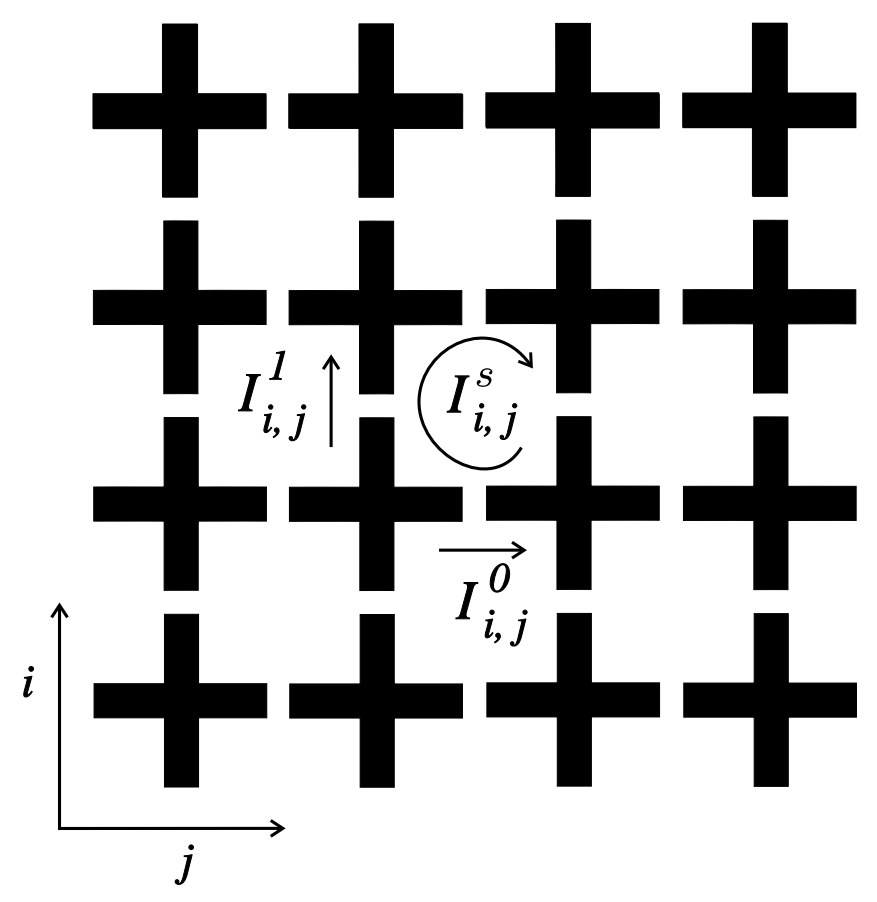


Fig.2a De Leo et al.

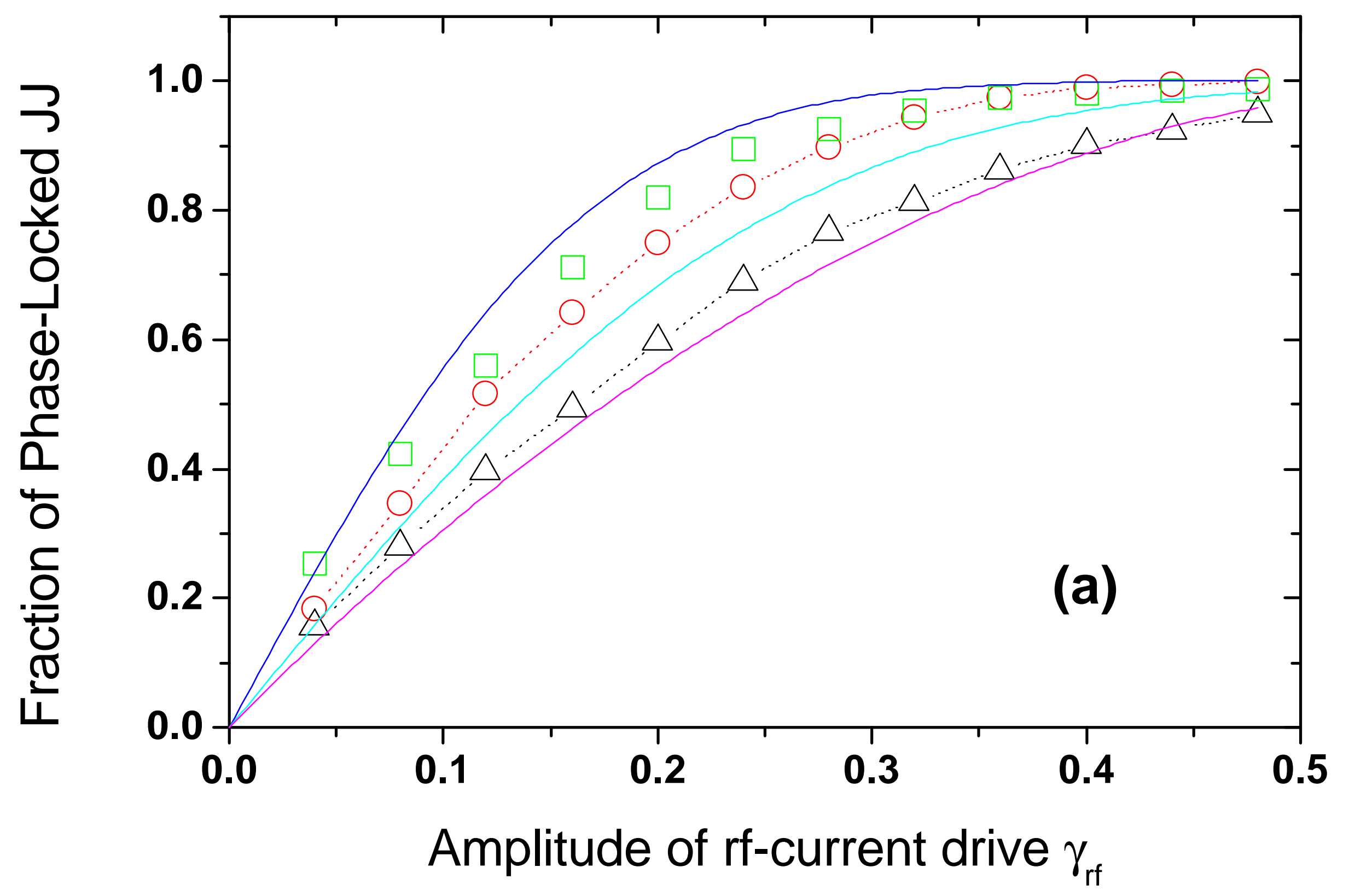


Fig.2b De Leo et al.

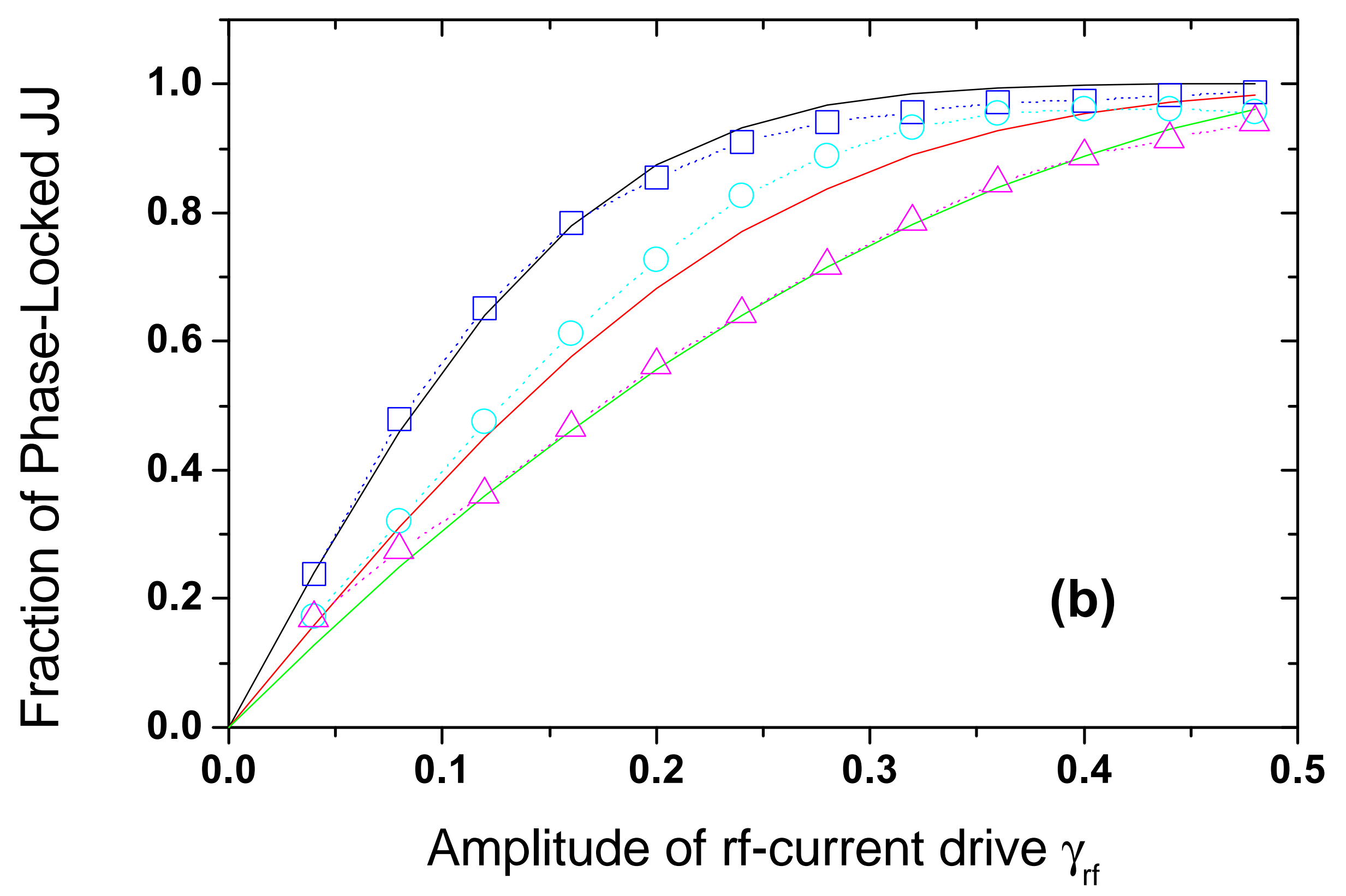


Fig.2c De Leo et al.

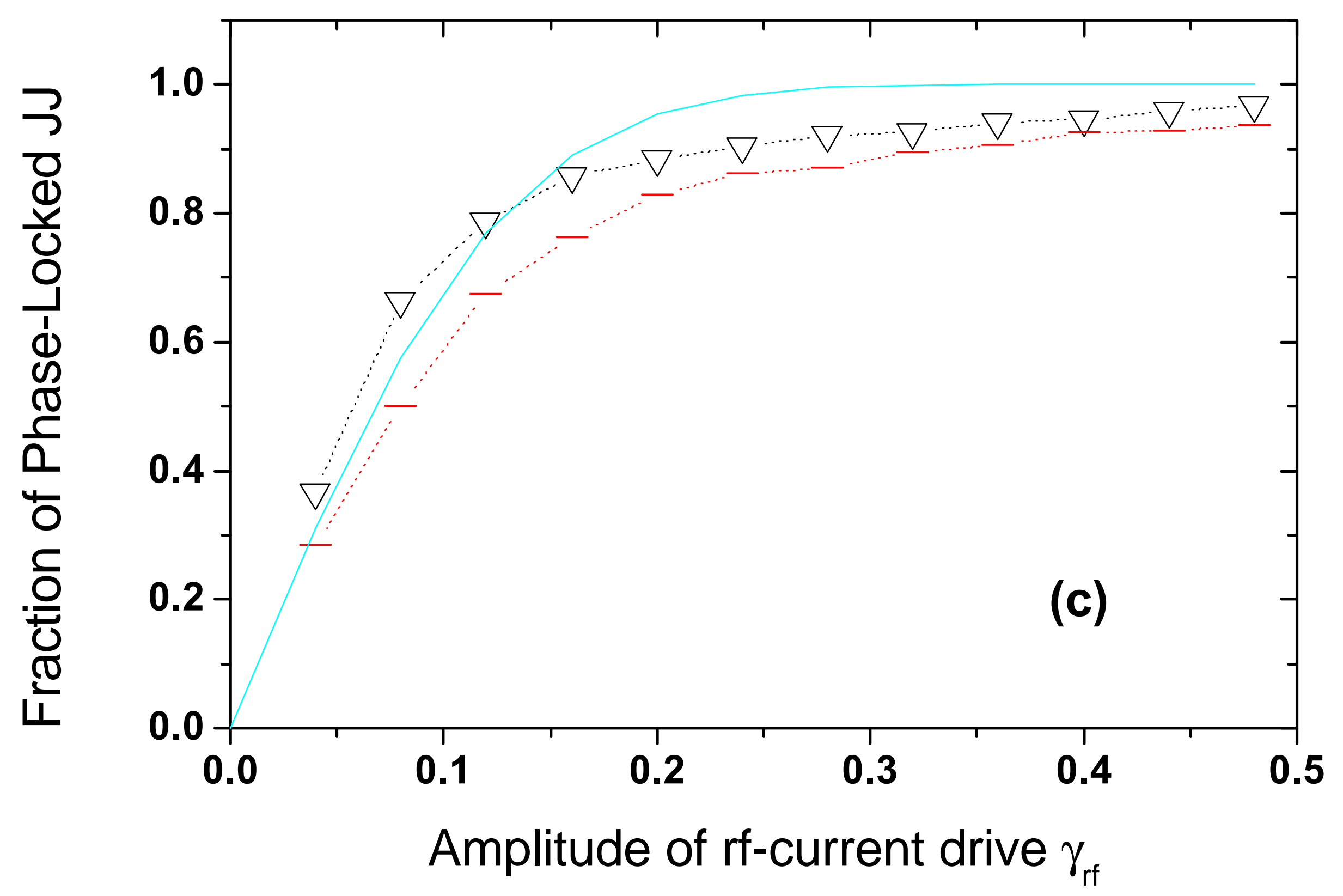


Fig.2d De Leo et al.

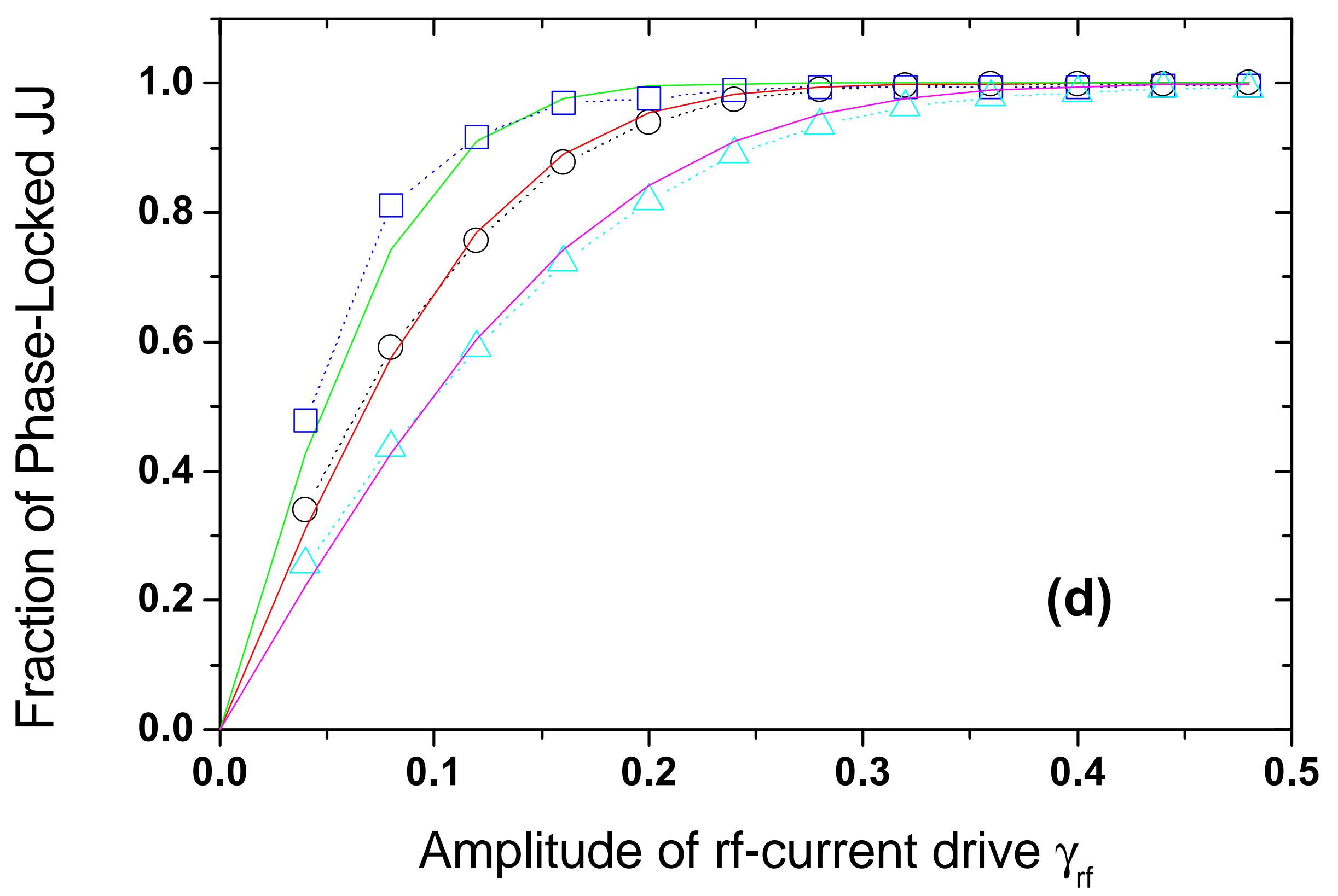


Fig.3a De Leo et al.

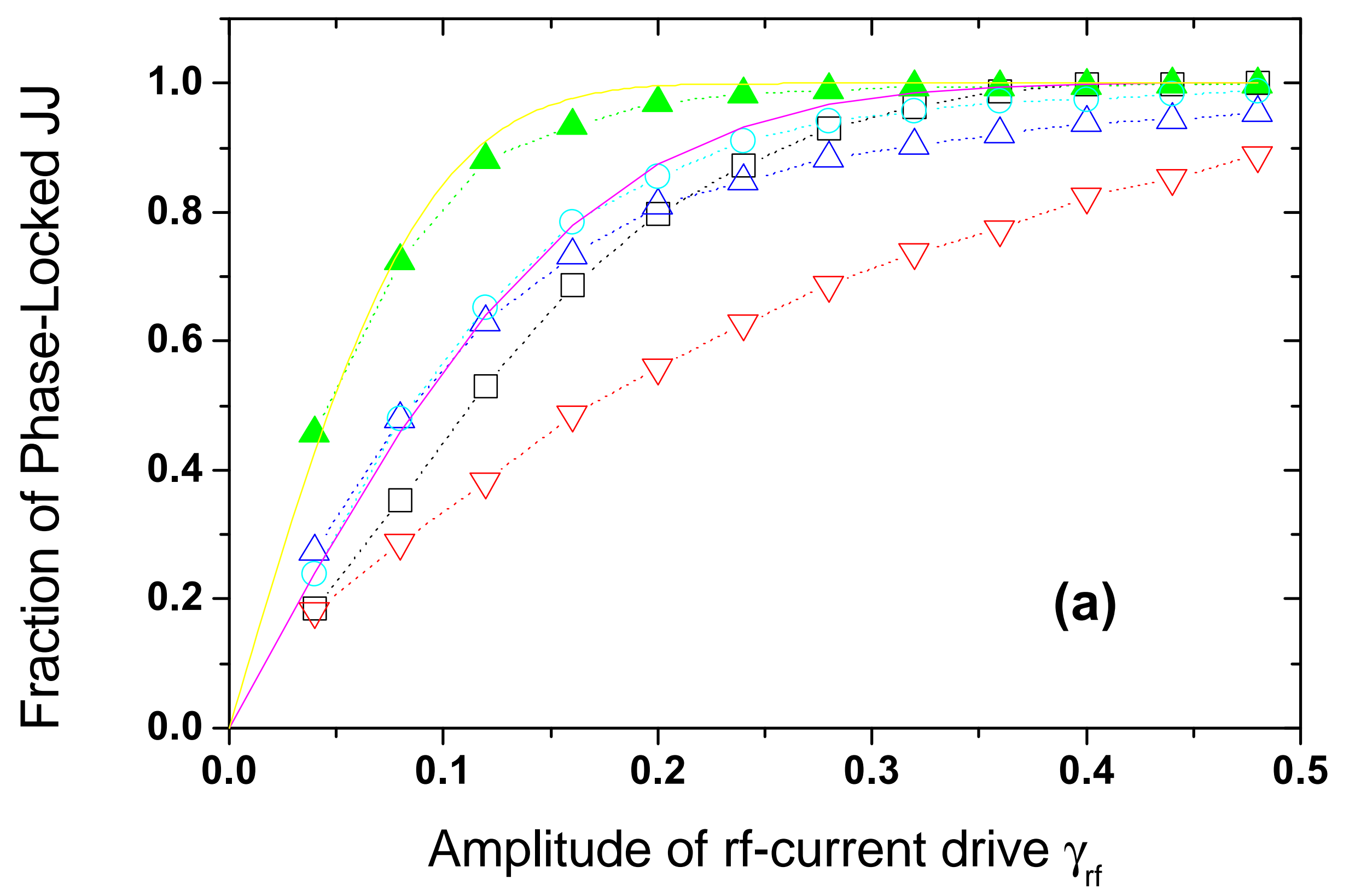


Fig.3b De Leo et al.

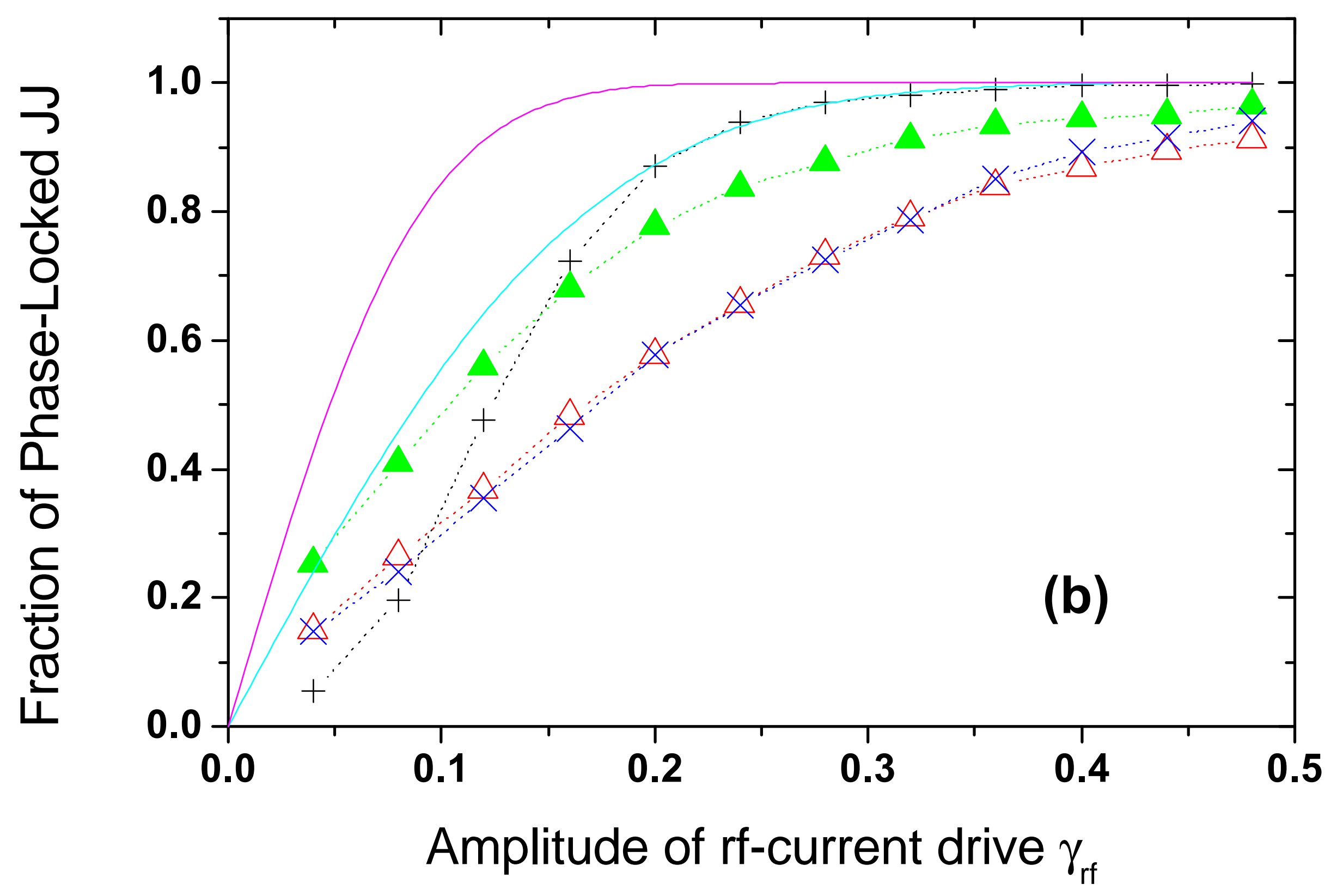


Fig.4a De Leo et al.

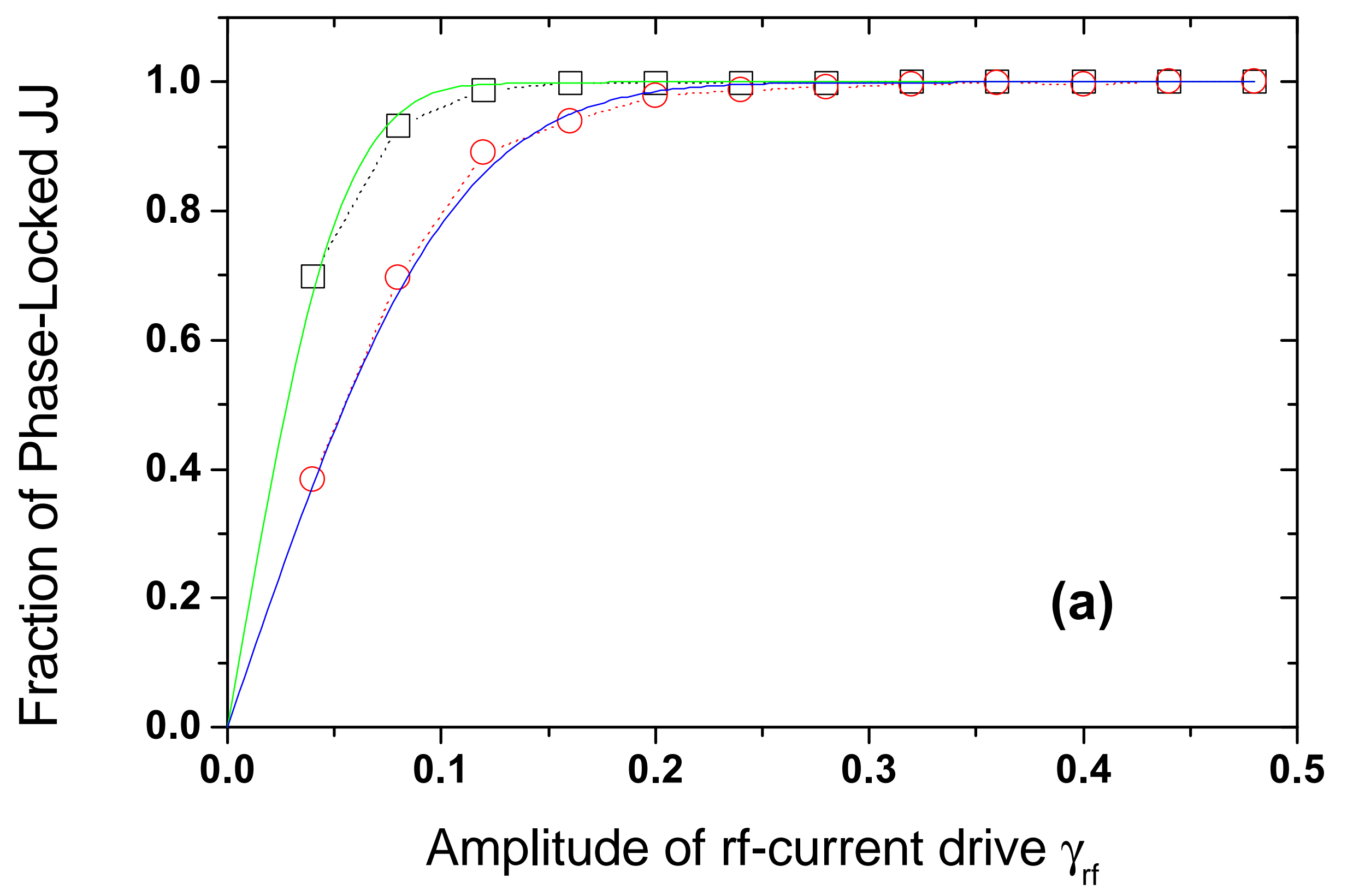


Fig.4b De Leo et al.

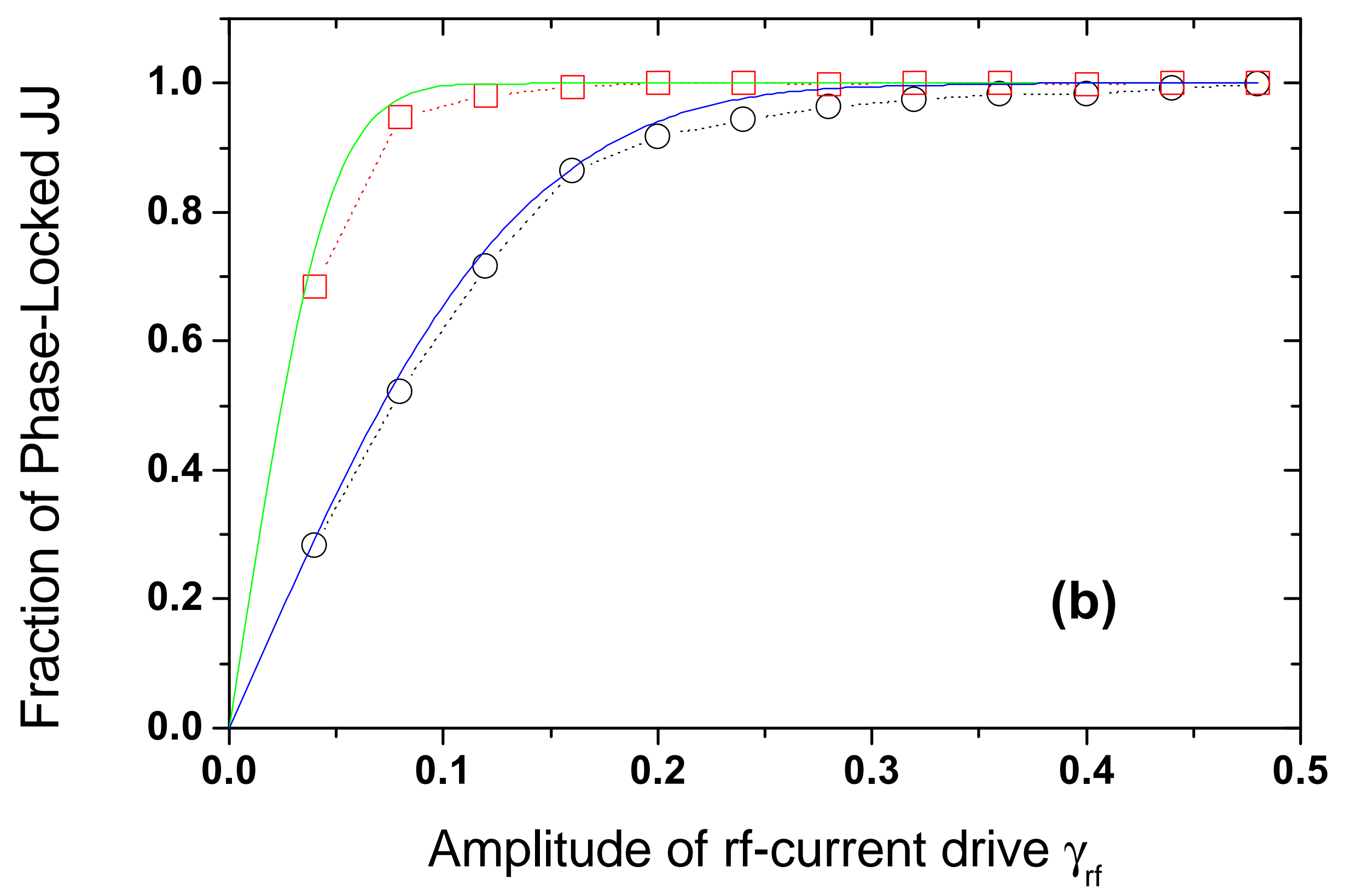


Fig.5 De Leo et al.

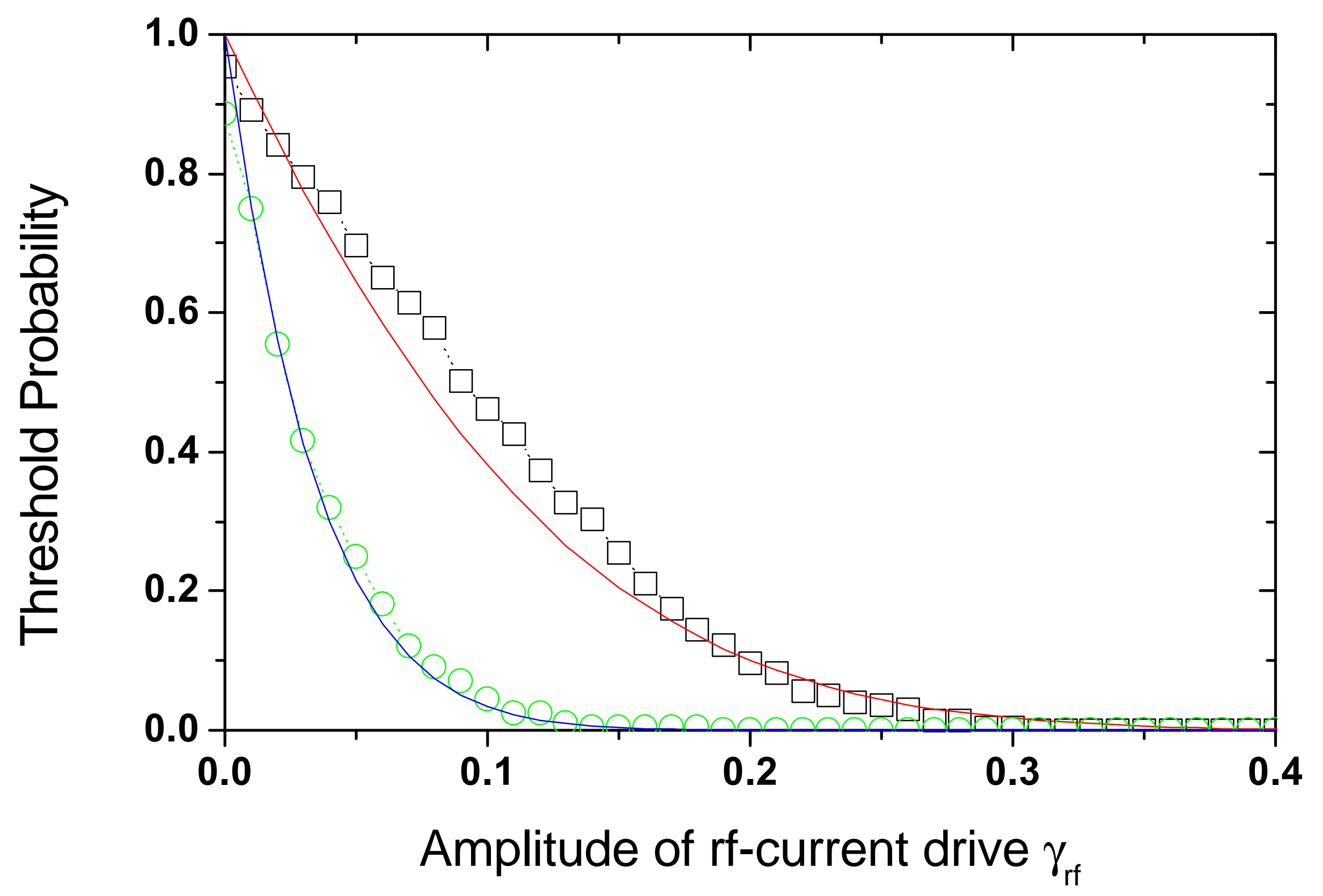

